\title{
Classification of Direct Kinematics to 3D3A Generalized Stewart Platform with Equilateral Triangle Platforms
}

\author{
Jiawen Zhang and Guifang Zhang* \\ College of Sciences, Beijing Forestry University, Beijing 100083, China \\ ${ }^{*}$ Corresponding author
}

\begin{abstract}
Generalized Stewart Platform is the extended form of $S$ tewart platform of parallel linkages, which is two rigid bodies connected with six constraints. The classification of direct kinematics to 3D3A generalized Stewart platform with equilateral triangle platforms based on symbolic computation is presented. The explicit conditions of the parameters for the generalized Stewart platforms to have a given number of real solutions are given while imposing three angular constraints and three distance constraints in sequence. The appropriate parameters can be selected according to the conditions of the parameters, such that the number of real solutions specified can be obtained.
\end{abstract}

Keywords-parallel linkages; direct kinematics; generalized Stewart platform; symbolic computation; Wu-Ritt characteristic set method

\section{INTRODUCTION}

The Stewart platform is a parallel manipulator consisting of two rigid bodies: a moving platform, or simply a platform, and a base, where the base and the platform are connected with six extensible legs ${ }^{[1]}$. A large portion of the work on Stewart platform is focused on the direct kinematics, i.e. determining the pose of the platform for a given set of lengths of the legs assuming that the position and orientation (pose) of the base are fixed. This problem is still not solved completely until now.

To find new and more practical parallel mechanisms for various purposes, the generalized Ste wart platform (abbr. GSP) consisting of two rigid bodies connected with six distance and/or angular constraints between six pairs of points, lines and/or planes in the base and the platform respectively is introduced, which could be considered as the most general form of parallel manipulators with six degrees of freedo ${ }^{[2]}$. Considering the types of six constraints imposed, there are 3850 types of GSPs totally [2]. In [2], direct kinematics to 3D3A GSP, i.e. the six constraints composed of three distance constraints (3D) and three angular constraints (3A), are studied with Wu-Ritt characteristic set method [3]. Because the distance constraint will not disrupt the angular constraint imposed previously ${ }^{[4]}$, three angular constraints and three distance constraints are imposed in sequence to find the closed form solutions to rotation matrix and translation matrix respectively, without considering the shapes of the platform and the base ${ }^{[2]}$. From then on, several authors have studied the kinematic properties of GSPs proposed in [2]. For example, the 3850 GSPs are reduced to 195 GSPs by disregarding the planar joints based on two criterions [5]. In [6], the strategies on direct kinematics to spatial GSP are discussed with graph theory based on the analysis of degrees of freedom of geometric primitives and degrees of constraints, Wu-Ritt characteristic set method [3] and complete discrimination system for polynomials [7], without considering the shapes of the platform and the base. For more research works related to spacial generalized Stewart platform, please refer to [5].

To find new and more practical planar parallel mechanisms for various purposes, the planar generalized Stewart platform consisting of two rigid bodies connected by three distance and/or angular constraints between three pairs of points and/or lines in the platform and the base is introduced ${ }^{[8]}$. There are 16 types of planar GSPs totally. In [9], the classification of direct kine matics to these planar GSPs and the parameter conditions of obtaining real solutions to 16 types of planar GSPs are presented.

In this paper, we discuss the classification of direct kine matics to 3D3A generalized Stewart platform, considering the shapes of the moving platform and the base. Both the moving platform and the base are equilateral triangle platforms. It is presented that the method of finding the explicit conditions of parameters for 3D3A generalized Stewart platform to have a given number of real solutions based on Wu-Ritt characteristic set method [3] and complete discrimination system for polynomials ${ }^{[7]}$.

\section{CLASSIFICATION OF DIRECT KINEMATICS TO 3D3A GENERALIZED STEWART PLATFORM}

The degree of freedom (abbr. DOF )of a rigid body is six in three-dimensional space, which consists of three rotation DOFs and three translation DOFs. Rigid body motion can be represented by $\boldsymbol{R}, \boldsymbol{T}$, where $\boldsymbol{R}=\left(r_{i j}\right)_{3 \times 3}(i, j=1,2,3)$ represents rotaion matrix $\boldsymbol{R}^{T} \boldsymbol{R}=\boldsymbol{R} \boldsymbol{R}^{T}=\boldsymbol{I},|\boldsymbol{R}|=1$, and $\boldsymbol{T}$ reprsents translation matrix in $\boldsymbol{R}^{\mathbf{3}}{ }^{[2]}$.

The pose of the platform of 3D3A GSP can be positioned in the two steps: imposing three angular constraints determine rotation matrix $\boldsymbol{R}$ firstly; imposing three angular constraints determine translation matrix $\boldsymbol{T}$ secondly.

Let the platform be equilateral triangle $\boldsymbol{P}_{\mathbf{1}} \boldsymbol{P}_{\mathbf{2}} \boldsymbol{P}_{\mathbf{3}}$ with side $a$ and the base equilateral triangle $\boldsymbol{B}_{\mathbf{1}} \boldsymbol{B}_{\mathbf{2}} \boldsymbol{B}_{\mathbf{3}}$ with side $b$. The local coordinates of points $\boldsymbol{P}_{\mathbf{1}}, \boldsymbol{P}_{\mathbf{2}}, \boldsymbol{P}_{\mathbf{3}}$ in the platform are 
$\boldsymbol{P}_{1}(0,0,0), \boldsymbol{P}_{2}(a, 0,0), \boldsymbol{P}_{3}\left(\frac{a}{2}, \frac{\sqrt{3}}{2} a, 0\right)$. The direction of $z$ axis of the local coordinates is upward and perpendicular to triangle $\boldsymbol{P}_{\mathbf{1}} \boldsymbol{P}_{\mathbf{2}} \boldsymbol{P}_{\mathbf{3}}$ with normal vector $\boldsymbol{s}_{\boldsymbol{p}}(0,0,1)$. The global coordinates of points $\boldsymbol{B}_{\mathbf{1}} \boldsymbol{B}_{\mathbf{2}} \boldsymbol{B}_{\mathbf{3}}$ in the base are $\boldsymbol{B}_{1}(0,0,0), \boldsymbol{B}_{2}(b, 0,0), \boldsymbol{B}_{3}\left(\frac{b}{2}, \frac{\sqrt{3}}{2} b, 0\right)$. The direction of $z$ axis of the global coordinates is upward and perpendicular to triangle $\boldsymbol{B}_{\mathbf{1}}, \boldsymbol{B}_{\mathbf{2}}, \boldsymbol{B}_{\mathbf{3}}$ with normal vector $\boldsymbol{s}_{b}(0,0,1)$.

\section{A. Imposing Angular Constraints}

There are two types of angular constraints between two vectors: parallel constraint and non- parallel constraint. Parallel constraint consists of two scalar equations, while non- parallel constraint consists of one scalar equation. Hence, there are two ways to add three angular constraints: a parallel constraint and a non-parallel cons traint, or three non-parallel constraints.

We take an example of imposing a parallel constraint between the platform and the base, and a non-parallel constraint between line $\boldsymbol{P}_{\mathbf{1}} \boldsymbol{P}_{\mathbf{2}}$ in the platform and line $\boldsymbol{B}_{\mathbf{1}} \boldsymbol{B}_{\mathbf{3}}$ in the base to demonstrate the method of classifying direct kine matics to 3D3A generalized Stewart platforms. Other cases can be classified in the similar way. The corresponding multivariate polynomials are shown as (1).

$$
\left\{\begin{array}{l}
\boldsymbol{R}^{T} \boldsymbol{R}=\boldsymbol{R} \boldsymbol{R}^{T}=\boldsymbol{I} \\
|\boldsymbol{R}|=1 \\
\boldsymbol{s}_{\boldsymbol{B}_{1} \boldsymbol{B}_{3}} \cdot \boldsymbol{R}_{\boldsymbol{P}_{1} \boldsymbol{P}_{2}}=d \\
r_{13}=0 \\
r_{23}=0
\end{array}\right.
$$

where $\quad \boldsymbol{s}_{\boldsymbol{B}_{1} \boldsymbol{B}_{3}}=\left(\frac{1}{2}, \frac{\sqrt{3}}{2}, 0\right) \quad, \quad \boldsymbol{S}_{\boldsymbol{P}_{1} \boldsymbol{P}_{2}}=(1,0,0)$ and $|d|=\left|\cos \left(\angle\left(\boldsymbol{s}_{B_{1} B_{3}}, \boldsymbol{R s}_{\boldsymbol{P}_{1} \boldsymbol{P}_{2}}\right)\right)\right|<1$.

(1) If $|d| \neq \frac{1}{2}$, characteristic sets (2) and (3) are obtained with $\mathrm{Wu}$-Ritt characteristic set method ${ }^{[3]}$.

$$
\left\{\begin{array}{l}
r_{33}+1=0 \\
4\left(r_{11}-2 d\right) r_{22}-4 r_{11} d-4 d^{2}+3=0 \\
r_{21}+r_{11}+2 d=0 \\
r_{12}+r_{11}-2 d=0 \\
4 r_{11}^{2}-4 d r_{11}+4 d^{2}-3=0
\end{array}\right.
$$

$$
\left\{\begin{array}{l}
r_{33}-1=0 \\
4\left(r_{11}-2 d\right) r_{22}+4 r_{11} d-4 d^{2}+3=0 \\
r_{21}+r_{11}+2 d=0 \\
r_{12}-r_{11}+2 d=0 \\
4 r_{11}^{2}-4 d r_{11}+4 d^{2}-3=0
\end{array}\right.
$$

where $r_{32}=r_{31}=0$. It is obviously that the number of real solutions to characteristic sets (2) and (3) depends on equation $4 r_{11}^{2}-4 d r_{11}+4 d^{2}-3=0$, which has two real solutions $r_{11}=\left(d \pm \sqrt{3\left(1-d^{2}\right)}\right) / 2$. Thus there are four real solutions to (1), and the corresponding rotation matrixes are as follows.

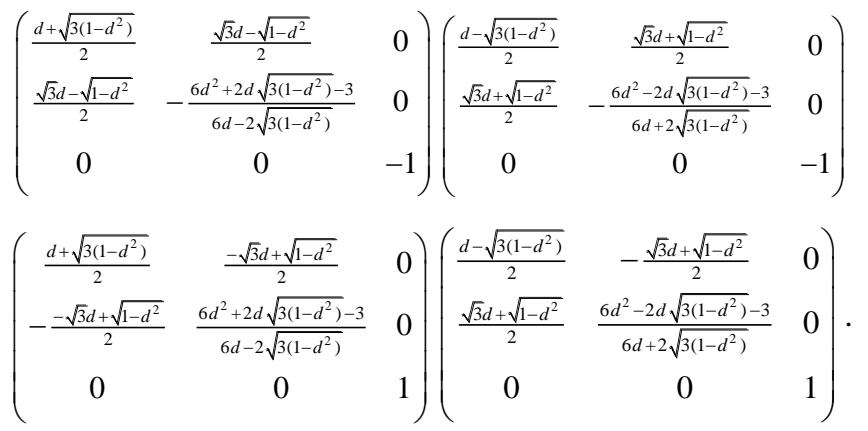

(2) If $d=\frac{1}{2}$, there are four solutions to (1), and the corresponding rotation matrixes are shown as follows.

$$
\left(\begin{array}{ccc}
-\frac{1}{2} & \frac{\sqrt{3}}{2} & 0 \\
\frac{\sqrt{3}}{2} & \frac{1}{2} & 0 \\
0 & 0 & -1
\end{array}\right)\left(\begin{array}{ccc}
-\frac{1}{2} & -\frac{\sqrt{3}}{2} & 0 \\
\frac{\sqrt{3}}{2} & -\frac{1}{2} & 0 \\
0 & 0 & 1
\end{array}\right)\left(\begin{array}{ccc}
1 & 0 & 0 \\
0 & -1 & 0 \\
0 & 0 & -1
\end{array}\right)\left(\begin{array}{lll}
1 & 0 & 0 \\
0 & 1 & 0 \\
0 & 0 & 1
\end{array}\right) .
$$

(3) If $d=-\frac{1}{2}$, there are four solutions to (1), and the corresponding rotation matrixes are shown as follows.

$$
\left(\begin{array}{ccc}
\frac{1}{2} & \frac{\sqrt{3}}{2} & 0 \\
-\frac{\sqrt{3}}{2} & \frac{1}{2} & 0 \\
0 & 0 & 1
\end{array}\right)\left(\begin{array}{ccc}
\frac{1}{2} & -\frac{\sqrt{3}}{2} & 0 \\
-\frac{\sqrt{3}}{2} & -\frac{1}{2} & 0 \\
0 & 0 & -1
\end{array}\right)\left(\begin{array}{ccc}
-1 & 0 & 0 \\
0 & -1 & 0 \\
0 & 0 & 1
\end{array}\right)\left(\begin{array}{ccc}
-1 & 0 & 0 \\
0 & 1 & 0 \\
0 & 0 & -1
\end{array}\right) \text {. }
$$

\section{B. Imposing Distance Constaints}

The platform can only do translational motion after removing three rotational degrees of freedom by imposing three angular constraints. Hence, the translation matrix can be obtained once the exact global coordinate of orig in of local coordinate in the platform can be confirmed by imposing three distance constraints. The pose of the platform can be positioned by rotating it according to rotation matrix and translating it according to translation matrix. All of the loci of the points that satisfy a distance constraint are called translational space after three rotational degrees of freedom are removed. The translational spaces of distance constraints are plane, sphere or cylinder assuming that three rotational degrees of freedom are removed previously ${ }^{[2]}$. The problem of imposing three distance constraints can be classified into 10 cases according to the shape of the translational spaces ${ }^{[2]}$. 
We take an example of imposing three distance constraint between points in the platform and the base to demonstrate the method of classifying direct kine matics to generalized Stewart platforms. Other cases can be classified in the similar way.

After imposing three angular and threes distance constraints, the global coordinates of points $\boldsymbol{P}_{1}(0,0,0)$, $\boldsymbol{P}_{2}(a, 0,0), \boldsymbol{P}_{3}\left(\frac{a}{2}, \frac{\sqrt{3}}{2} a, 0\right)$ in the platform are transformed and denoted by $\boldsymbol{P}_{1}^{\prime}=(x, y, z), \quad \boldsymbol{P}_{2}^{\prime}=\left(a r_{11}+x, a r_{12}+y, a r_{13}+z\right)$ and $\boldsymbol{P}_{3}^{\prime}=\left(\frac{a}{2} r_{11}+\frac{\sqrt{3}}{2} a r_{12}+x, \frac{a}{2} r_{21}+\frac{\sqrt{3}}{2} a r_{22}+y, \frac{a}{2} r_{31}+\frac{\sqrt{3}}{2} a r_{32}+z\right)$.

Let three distance constraints be

$$
\left|\boldsymbol{P}_{1}^{\prime} \boldsymbol{B}_{1}\right|^{2}=d_{1},\left|\boldsymbol{P}_{2}^{\prime} \boldsymbol{B}_{2}\right|^{2}=d_{2},\left|\boldsymbol{P}_{3}^{\prime} \boldsymbol{B}_{3}\right|^{2}=d_{3} .
$$

The corresponding equation systems are shown as (4).

$$
\left\{\begin{array}{l}
x^{2}+y^{2}+z^{2}-d_{1}=0 \\
\left(a r_{11}+x-b\right)^{2}+\left(a r_{12}+y\right)^{2}+\left(a r_{13}+z\right)^{2}-d_{2}=0 \\
\left(\frac{a}{2} r_{11}+\frac{\sqrt{3}}{2} a r_{12}+x-\frac{b}{2}\right)^{2}+\left(\frac{a}{2} r_{21}+\frac{\sqrt{3}}{2} a r_{22}+y-\frac{\sqrt{3}}{2} b\right)^{2} \\
+\left(\frac{a}{2} r_{31}+\frac{\sqrt{3}}{2} a r_{32}+z\right)^{2}-d_{3}=0
\end{array}\right.
$$

where $d_{1}>0, d_{2}>0, d_{3}>0$.

(1) If $-3 a r_{13} r_{22}+\sqrt{3} a r_{31} r_{12}-\sqrt{3} a r_{13} r_{21}+3 a r_{12} r_{32}+3 r_{13} b \neq 0$, characteristic set (5) can be obtained with Wu-Ritt characteristic set method ${ }^{[3]}$.

$$
\left\{\begin{array}{l}
z+c_{11} x+c_{12}=0 \\
y+c_{13} x+c_{14}=0 \\
c_{15} x^{2}+c_{16} x+c_{17}=0
\end{array}\right.
$$

where each $\mathrm{c}_{\mathrm{ij}}$ is composed of $a, b, r_{i j}$ and $d_{i}$. The number of real solutions to characteristic set (5) depends on the quadratic equation. We can obtain one or two real solutions and select appropriate parameters for specified number of real solutions according to the discriminant of the quadratic equation.

(2) If $-3 a r_{13} r_{22}+\sqrt{3} a r_{31} r_{12}-\sqrt{3} a r_{13} r_{21}+3 a r_{12} r_{32}+3 r_{13} b=0$,

five different characteristic sets with different parameter conditions can be obtained with Wu-Ritt characteristic set method [3]. Each of the characteristic sets is composed of two linear equations and one quadratic equation. Although each of the characteristic sets is similar as characteristic set (5), the main variable of the quadratic equation is different from (5). The number of real solutions to each characteristic set also depends on the discriminant of the quadratic equation.

\section{EXAMPLE}

\section{A. Imposing Angular Constraints}

Three angular constraints are three non-parallel constraints between three pairs of lines in the platform and the base, i.e. $\cos \left(\angle\left(\boldsymbol{s}_{\boldsymbol{B}_{2} \boldsymbol{B}_{3}}, \boldsymbol{R}_{\boldsymbol{P}_{2} \boldsymbol{P}_{3}}\right)\right)=t(|t|<1) \quad, \quad \angle\left(\boldsymbol{s}_{\boldsymbol{B}_{1} \boldsymbol{B}_{2}}, \boldsymbol{R}_{\boldsymbol{P}_{\boldsymbol{P}_{2} \boldsymbol{P}_{2}}}\right)=\frac{\pi}{2}$, $\angle\left(\boldsymbol{s}_{\boldsymbol{B}_{1} \boldsymbol{B}_{3}}, \boldsymbol{R} \boldsymbol{s}_{\boldsymbol{P}_{1} \boldsymbol{P}_{3}}\right)=\frac{\pi}{3}$, where $\boldsymbol{s}_{B_{i} B_{j}}$ and $\boldsymbol{s}_{\boldsymbol{P}_{i} \boldsymbol{P}_{j}}$ are unit vectors of lines $\boldsymbol{B}_{i} \boldsymbol{B}_{j}$ and $\boldsymbol{P}_{i} \boldsymbol{P}_{j}$ respectively. The corresponding multivariate polynomials are shown as (6).

$$
\left\{\begin{array}{l}
\boldsymbol{R}^{T} \boldsymbol{R}=\boldsymbol{R} \boldsymbol{R}^{T}=\boldsymbol{I} \\
|\boldsymbol{R}|=1 \\
\boldsymbol{s}_{B_{1} B_{2}} \cdot \boldsymbol{R} \boldsymbol{s}_{P_{1} P_{2}}=0 \\
\boldsymbol{s}_{B_{1} B_{3}} \cdot \boldsymbol{R} \boldsymbol{s}_{P_{1} P_{3}}=\frac{1}{2} \\
\boldsymbol{s}_{B_{2} B_{3}} \cdot \boldsymbol{R}_{\boldsymbol{P}_{2} P_{3}}=t
\end{array}\right.
$$

(1) If $t \neq-\frac{1}{2}$ and $16 t^{2}-8 t-5 \neq 0$, characteristic set (7) can be obtained with $\mathrm{Wu}$-Ritt characteristic set method.

$$
\left\{\begin{array}{l}
{\left[-9 \sqrt{3} \mathrm{r}_{12}^{2}-18(2 t-1) r_{12}-6 \sqrt{3}\left(2 t^{2}+2 t+1\right)\right] r_{33}} \\
+9(2 t-1) r_{12}^{3}+3 \sqrt{3}\left(8 t^{2}-8 t+5\right) r_{12}^{2}+ \\
12\left(2 t^{3}-3 t^{2}+3 t-1\right) r_{12}+\sqrt{3}\left(16 t^{2}-16 t-5\right)=0 \\
\sqrt{3} r_{32}+\left(\sqrt{3} r_{12}+2 t-1\right) r_{13}=0 \\
(2 t+1) r_{31}-\left[3 r_{12}^{2}+2 \sqrt{3}(2 t-1) r_{12}+2\left(t^{2}-2 t-1\right)\right] r_{13}=0 \\
(2 t+1) r_{23}-\left[3 r_{12}^{2}+2 \sqrt{3}(2 t-1) r_{12}+2\left(t^{2}-2 t-1\right)\right] r_{12} r_{13}=0 \\
3 r_{22}-2 t-1=0 \\
\sqrt{3} r_{21}+\sqrt{3} r_{12}+2 t-1=0 \\
{\left[18 \sqrt{3}(2 t-1) r_{12}^{3}+45(2 t-1)^{2} r_{12}^{2}+6 \sqrt{3}\left(8 t^{2}-8 t-1\right)(2 t-1) r_{12}\right.} \\
\left.+24\left(2 t^{4}-4 t^{3}+2 t^{2}+t\right)-3\right] r_{13}^{2}+3\left(4 t^{2}+3 t+1\right) r_{12}^{2} \\
+2 \sqrt{3}\left(8 t^{3}+4 t^{2}-2 t-1\right) r_{12}+2\left(8 t^{4}-10 t^{2}-6 t-1\right)=0 \\
9 r_{12}^{4}+6 \sqrt{3}(2 t-1) r_{12}^{3}+3\left(4 t^{2}-4 t-5\right) r_{12}^{2}-6 \sqrt{3}(2 t-1) r_{12} \\
-16 t^{2}+8 t+5=0 \\
r_{11}=0
\end{array}\right.
$$

The number of real solutions to characteristic set (7) depends on both the seventh and the eighth equations.

The seventh equation is a quadratic equation of $r_{13}$. It has one real solution if $t=\frac{1}{2}$, and two real solutions if its discriminant is positive.

The eighth equation is a quartic equation of $r_{12}$. The corresponding $\mathrm{d}$ iscrimination sequence based on the complete discrimination system for polynomials ${ }^{[7]}$ is as follows. 


$$
\left\{\begin{array}{l}
D_{1}=1>0 \\
D_{2}=4(2 t-1)^{2}+48>0 \\
\left.D_{3}=\left[\left(2 t-\frac{1}{2}\right)^{2}+\frac{3}{4}\right]\left[(2 t-1)^{2}+12\right)\right]>0 \\
D_{4}=\left(4 t^{2}-12 t-15\right)\left(4 t^{2}+4 t-7\right)\left(4 t^{2}-2 t+1\right)^{2}
\end{array}\right.
$$

According to the revised sign tables of discriminant sequence for a univariate polynomial of degree four ${ }^{[7]}$, the following conditions are obtained.

(a) If $D_{4}>0$, i.e. $\frac{3}{2}-\sqrt{6}<t<\sqrt{2}-\frac{1}{2}$, there are four real solutions.

(b) If $D_{4}=0$, i.e. $t=\sqrt{2}-\frac{1}{2}$ or $t=\frac{3}{2}-\sqrt{6}$, there are three real solutions.

(c) If $D_{4}<0$, i.e. $\sqrt{2}-\frac{1}{2}<t<1$ or $-1<t<\frac{3}{2}-\sqrt{6}$, there are two real solutions.

The appropriate parameter $d_{1}$ can be selected to obtain the desired number of real solution according to above conditions.

For example, if $t=\frac{1}{2}$, there are four real solutions to (7) and the rotation matrix are as follows.

$$
\left(\begin{array}{ccc}
0 & \frac{\sqrt{3}}{3} & \pm \frac{\sqrt{6}}{3} \\
\frac{-\sqrt{3}}{3} & \frac{2}{3} & \mp \frac{\sqrt{2}}{3} \\
\mp \frac{\sqrt{6}}{3} & \mp \frac{\sqrt{2}}{3} & \frac{1}{3}
\end{array}\right)\left(\begin{array}{ccc}
0 & \frac{-\sqrt{3}}{3} & \pm \frac{\sqrt{6}}{3} \\
\frac{\sqrt{3}}{3} & \frac{2}{3} & \pm \frac{\sqrt{2}}{3} \\
\mp \frac{\sqrt{6}}{3} & \pm \frac{\sqrt{2}}{3} & \frac{1}{3}
\end{array}\right)
$$

$$
\text { If } t=-\frac{1}{2} \text {, there are four real solutions to (6) and the }
$$
rotation matrix are as follows.

$$
\left(\begin{array}{ccc}
0 & 1 & 0 \\
\frac{2}{\sqrt{3}}-1 & 0 & \pm 2 \sqrt{\frac{\sqrt{3}-1}{3}} \\
\pm 2 \sqrt{\frac{\sqrt{3}-1}{3}} & 0 & 1-\frac{2}{\sqrt{3}}
\end{array}\right)\left(\begin{array}{ccc}
0 & \frac{2}{\sqrt{3}}-1 & \pm 2 \sqrt{\frac{\sqrt{3}-1}{3}} \\
1 & 0 & 0 \\
0 & \pm 2 \sqrt{\frac{\sqrt{3}-1}{3}} & 1-\frac{2}{\sqrt{3}}
\end{array}\right)
$$

$$
\text { If } t=\frac{1+\sqrt{6}}{4} \text {, i.e. } 16 d_{1}^{2}-8 d_{1}-5=0 \text {, there are two real }
$$

solutions to (6) and the rotation matrix are as follows.

$$
\left(\begin{array}{ccc}
0 & \frac{\sqrt{3}}{6}-\frac{\sqrt{2}}{2} & -\frac{\sqrt{6}+3}{6} \\
0 & \frac{\sqrt{6}+3}{6} & \frac{\sqrt{3}}{6}-\frac{\sqrt{2}}{2} \\
1 & 0 & 0
\end{array}\right)\left(\begin{array}{ccc}
0 & \frac{\sqrt{3}}{6}-\frac{\sqrt{2}}{2} & \frac{\sqrt{6}+3}{6} \\
0 & \frac{\sqrt{6}+3}{6} & \frac{\sqrt{2}}{2}-\frac{\sqrt{3}}{6} \\
-1 & 0 & 0
\end{array}\right)
$$

(4) If $t=\frac{1-\sqrt{6}}{4}$, i.e. $16 d_{1}^{2}-8 d_{1}-5=0$, there are two real solutions to (6) and the rotation matrix are as follows.

$$
\left(\begin{array}{ccc}
0 & \frac{\sqrt{3}}{6}+\frac{\sqrt{2}}{2} & \frac{\sqrt{6}-3}{6} \\
0 & \frac{3-\sqrt{6}}{6} & \frac{\sqrt{3}}{6}+\frac{\sqrt{2}}{2} \\
1 & 0 & 0
\end{array}\right)\left(\begin{array}{ccc}
0 & \frac{\sqrt{3}}{6}+\frac{\sqrt{2}}{2} & -\frac{\sqrt{6}-3}{6} \\
0 & \frac{3-\sqrt{6}}{6} & -\left(\frac{\sqrt{3}}{6}+\frac{\sqrt{2}}{2}\right) \\
-1 & 0 & 0
\end{array}\right)
$$

\section{B. Imposing Distance Constraints}

Three distance constraints are: the distance between point $\boldsymbol{P}_{\mathbf{3}}^{\prime}$ in the platform and plane $\boldsymbol{B}_{\mathbf{1}} \boldsymbol{B}_{\mathbf{2}} \boldsymbol{B}_{\mathbf{3}}$ in the base is $\sqrt{t_{1}}$ (the translational space is plane), the distance between point $\boldsymbol{P}_{2}^{\prime}$ in the platform and point $\boldsymbol{B}_{2}$ in the base is $\sqrt{t_{2}}\left(t_{2}>0\right)$ (the translational space is sphere), and the distance between point $\boldsymbol{P}_{\mathbf{1}}^{\prime}$ in the platform and line $\boldsymbol{B}_{\mathbf{1}} \boldsymbol{B}_{\mathbf{2}}$ in the base is $\sqrt{t_{3}}$ (the translational space is cylinder). The corresponding nonlinear multivariate polynomials are shown as (8).

$\left\{\begin{array}{l}\left|\frac{a}{2} r_{31}+\frac{a \sqrt{3}}{2} r_{32}+z\right|-\sqrt{t_{1}}=0 \\ \left(a r_{11}+x-b\right)^{2}+\left(a r_{12}+y\right)^{2}+\left(a r_{13}+z\right)^{2}-t_{2}=0 \\ y^{2}+z^{2}-t_{3}=0\end{array}\right.$

Now characteristic set (9) can be obtained with Wu-Ritt characteristic set method, where $t_{4}= \pm \sqrt{t_{1}}$.

$\left\{\begin{array}{l}x^{2}+2\left(a r_{11}-b\right) x+2 a r_{12} y+\left(r_{11}^{2}+r_{12}^{2}+r_{13}^{2}\right) a^{2} \\ -\left(\sqrt{3} r_{32}-r_{31}\right) r_{13} a^{2}+2\left(r_{13} t_{4}-r_{11} b\right) a+b^{2}-t_{2}+t_{3}=0 \\ 4 y^{2}+\left(r_{31}+\sqrt{3} r_{32}\right)^{2} a^{2}-4\left(r_{31}+\sqrt{3} r_{32}\right)+4 t_{4}{ }^{2}-4 t_{3}=0 \\ 2 z+a \sqrt{3} r_{32}+a r_{31}-2 t_{4}=0\end{array}\right.$

The number of real solutions depends on parameters $a, b, t_{2}, t_{3}, t_{4}$ once the rotation matrix $\left(\mathrm{r}_{i j}\right)_{3 \times 3}$ is given.

For example, let the rotation matrix $\left(\mathrm{r}_{i j}\right)_{3 \times 3}$ be the first rotation matrix $\left(t=\frac{1}{2}\right)$ of (7), i.e.

$\left\{r_{11}=0, r_{12}=-r_{21}=\frac{\sqrt{3}}{3}, r_{13}=-r_{31}=\frac{\sqrt{6}}{3}, r_{22}=\frac{2}{3}, r_{23}=r_{32}=\frac{-\sqrt{2}}{3}, r_{33}=\frac{1}{3}\right\}$.

Parameter conditions to (9) are $t_{3} \geq\left(\frac{\sqrt{6}}{3} a+t_{4}\right)^{2}$ and

$2 \sqrt{3} a \sqrt{t_{3}-\left(\frac{\sqrt{6}}{3} a+t_{4}\right)^{2}} \leq\left[3\left(t_{2}-t_{3}\right)-7 a^{2}-2 \sqrt{6} a t_{4}\right]$

or

$2 \sqrt{3} a \sqrt{t_{3}-\left(\frac{\sqrt{6}}{3} a+t_{4}\right)^{2}} \geq-\left[3\left(t_{2}-t_{3}\right)-7 a^{2}-2 \sqrt{6} a t_{4}\right]$.

\section{CONCLUSION}

The problem to classify direct kinematics to 3D3A generalized Stewart platform when both the platform and the base are equilateral triangles is studied in this paper. The experiment results indicate the effectiveness and practicability of the analytical method based on Wu-Ritt characteristic set method and the complete discrimination system for polynomials. Due to space constraints, only a few of them can be listed here. Other cases imposing three angle constraints and three distance constraints can be studied according to the similar method.

The research works of this paper have been implemented on the computer algebra system Maple. The software package includes interactive angular and distance constraint parameter input, solving transfer matrix to position vertex coordinates of the platform, and graphic display the pose of the platform. 


\section{ACKNOWLEDGMENT}

This research was financially supported by the National Natural Science Foundation of China (No. 11501032, 60803099) and the Fundamental Research for the Central Universities (No. 2015ZCQ-LY-01).

\section{REFERENCE}

[1] B.Dasgupa, T.S. Mruthyunjaya, The Stewart Platform Manipulator: A Review[J]. Mechanism and Machine Theory, 2000, 35(1), pp. 15-40.

[2] X.S. Gao, D.L. Lei, Q. Z. Liao, G.F. Zhang, Generalized Stewart-Gough Platforms and Their Direct Kinematics[J]. IEEE Transactions on Robotics, 2005, 21(2), pp.141-151.

[3] W.T. Wu, Basic Principles of Mechanical Theorem Proving in geometries[M]. Springer, Berlin, 1995.

[4] A.V. Kumar, L. Yu, Sequential Constraint Imposition for Dimensiondriven Solid Models[J]. Computer Aided Design, 2001, 33(6), pp. 475486.

[5] M. Toz, S. Kucuk, Parallel Manipulator Software Tool for Design, Analysis, and Simulation of 195 GSP Mechanisms[J]. Computer Applications in Engineering Education, 2015, 6(11), pp. 931-946.

[6] G. F. Zhang, J.W. Zhang, L.N. Zhao, Strategies on Direct Kinematics to Generalized Stewart Platforms[C]. 2016 International Conference on Electrical Engineering and Aut omation, 2016, pp. 451-456.

[7] L.Yang, X.R. Hou, Z.B. Zeng, A Complete Discrimination System for Polynomials[J]. Science in China (Series E), 1996, 39(6), pp. 628-646.

[8] G. F. Zhang, X.S. Gao, Planar Generalized Stewart Platforms and Their Direct Kinematics[C]. International Workshop on Automated Deduction in Geometry, 2004, pp. 198-211.

[9] G. F. Zhang, Classification of Direct Kinematics to Planar Generalized Stewart Platforms [J]. Computational Geometry: Theory and Applications, 2012, 45(8), pp. 458-473. 